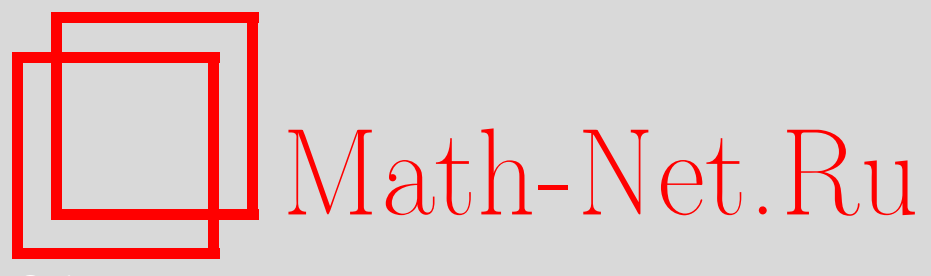

М. А. Черепнев, Некоторые свойства болыших простых делителей чисел вида $p-1$, Матем. заметки, 2006, том 80, выпуск 6, 920-925

DOI: https://doi.org/10.4213/mzm3367

Использование Общероссийского математического портала Math-Net.Ru подразумевает, что вы прочитали и согласны с пользовательским соглашением http://www . mathnet.ru/rus/agreement

Параметры загрузки:

IP : 52.6 .47 .48

26 апреля 2023 г., 18:14:49

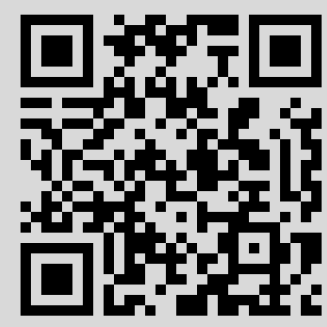




\section{НЕКОТОРЫЕ СВОЙСТВА}

\section{БОЛЬШИХ ПРОСТЫХ ДЕЛИТЕЛЕЙ ЧИСЕЛ ВИДА $p-1$}

\section{М. А. Черепнёв}

Основной результат статьи устанавливает, что доля простых чисел $p \leqslant x$ c условием, что $p-1$ имеет простой делитель $q>\exp (\ln x / \ln \ln x)$ и количество простых делителей $q-1$ существенно отличается от $\ln \ln (x / n)$, где $n=(p-1) / q$, стремится к нулю с ростом $x$.

Библиография: 6 названий.

Для оценки стойкости многих криптографических протоколов требуется сравнить стойкости задач дискретного логарифмирования и Диффи-Хеллмана [1]. В работе [2] эта задача сведена к оценке следующей теоретико-числовой функции. Пусть

$$
m=\prod_{i=1}^{r} p_{i}^{\alpha_{i}} .
$$

Рассмотрим разложение на простые множители чисел $p_{i}-1, i=1, \ldots, r$. Из каждого из этих простых вычтем единицу и снова разложим на простые, и т.д. Получившееся разветвление назовем деревом числа $\mathrm{m}$, а встречающиеся в нем простые числа - его узлами. Обозначим $s=s(m)$ длину наибольшей ветви дерева числа $m$.

Для оценки $s(m)$ для почти всех $m$ можно использовать результаты о количестве различных простых делителей чисел вида $p-1$. Оценка снизу для этого количества дает оценку сверху для самих простых делителей, которую в дальнейшем можно использовать для оценки $s(m)$.

В своих работах Харди и Рамануджан [3], Туран [4] показали, что для всех за исключением $o(x)$ натуральных значений $n, n \leqslant x$, выполняются неравенства

$$
(1-\varepsilon) \ln \ln n<v(n)<(1+\varepsilon) \ln \ln n,
$$

где $v(n)$ - количество различных простых делителей числа $n$. Аналогичный результат для чисел вида $p-1$, где $p$ - простое число, получил Эрдёш [5]. В данной заметке похожий результат получен для количества простых делителей чисел $q-1$, где $q-$ большой простой делитель числа $p-1$.

Заметим, что ввиду очевидной оценки $s(n) \leqslant \log _{2} n$ маленькие простые делители не оказывают влияния на величину $s(m)$.

Итак, пусть $N(M)$ - количество элементов в множестве $M$.

(C) М. А. ЧЕРепнЁв, 2006 
Tеорема 1. Для любого $\varepsilon>0$

$N\left(p \leqslant x \mid \begin{array}{c}\text { для всех простых } q>e^{(\ln x) /(\ln \ln x)}: \quad \frac{p-1}{q}=n \in \mathbb{N}, \\ \text { выполнено } v(q-1) \in\left[(1-\varepsilon) \ln \ln \frac{x}{n},(1+\varepsilon) \ln \ln \frac{x}{n}\right]\end{array}\right)=(1+o(1)) \frac{x}{\ln x}$.

Докажем сначала следующую лемму.

ЛЕмма 1. Найдутся абсолютные положительные константы $C_{1}, C_{2}$ такие, что для любъх $k, n \in \mathbb{N}$

$$
\begin{aligned}
N(p & \left.\leqslant x \mid \frac{p-1}{n}=q-\text { nростоe, } v(q-1)=k\right) \\
& <C_{1} \frac{x(n+1)\left(\ln \ln (x / n)+C_{2}\right)^{k+2}}{(k-1) ! \varphi(n(n+1)) \ln ^{3}(x / n)}+o\left(\frac{x}{n(\ln (x / n))^{(1 / 2) \ln \ln \ln (x / n)}}\right)
\end{aligned}
$$

при $x / n \rightarrow \infty$, причем о $(\cdot)$ не зависит от $k$ и от $n$.

ДокАЗАТЕЛЬСТво. Обозначим

$$
\frac{p-1}{n}=q \leqslant \frac{x-1}{n}=f(x) .
$$

Рассмотрим множество $M_{1}$ чисел $p \leqslant x$, для которых $(p-1) / n-$ простое и любой простой делитель числа $(p-1) / n-1$ меньше $y(f(x))$, где $y(z)=e^{(\ln z) /(\ln \ln z)}$. Обозначим через $N(x, y)$ число натуральных чисел, не превосходящих $x$, все простые делители которых не превосходят $y$. Для оценки этой величины применим известную лемму Ранкина [6; гл. 5, §5, лемма 5.2]. Получим

$$
\begin{aligned}
N\left(M_{1}\right) & <N\left(q-\text { простое } \mid q \leqslant f(x),\left\{\begin{array}{l}
r \mid q-1 \\
r-\text { простое }
\end{array} \Longrightarrow r \leqslant y(f(x))\right)\right. \\
& \leqslant N\left(m-\text { натуральное } \mid m \leqslant f(x),\left\{\begin{array}{l}
r \mid m \\
r-\text { простое }
\end{array} \Longrightarrow r \leqslant y(f(x))\right)\right. \\
& \leqslant N(f(x), y(f(x))) \\
& <f(x) \exp \left(-\frac{\ln _{2}\left((\ln f(x)) /\left(\ln _{2} f(x)\right)\right) \ln _{2} f(x)}{\ln f(x)} \ln f(x)+\ln \frac{\ln f(x)}{\ln _{2} f(x)}(1+o(1))\right) \\
& =o\left(f(x) \exp \left(-\frac{1}{2} \ln _{3} f(x) \ln _{2} f(x)\right)\right)=o\left(\frac{x}{n} \exp \left(-\frac{1}{2} \ln _{3} \frac{x}{n} \ln _{2} \frac{x}{n}\right)\right) .
\end{aligned}
$$

Здесь и далее используется обозначение

$$
\ln _{k}(x)=\underbrace{\ln \ln \cdots \ln }_{k \text { раз }}(x) .
$$

Полученная оценка показывает, что мощность множества $M_{1}$ не больше остаточного члена в формулировке леммы.

Рассмотрим множество $M_{2}$ чисел $p \leqslant x$, для которых $(p-1) / n$ - простое и наибольший простой делитель числа $(p-1) / n-1$ входит в разложение этого числа в 
степени не меньше 2. Тогда для $p \in M_{2} \backslash M_{1}$ число $(p-1) / n-1$ делится на квадрат числа, не меньшего, чем $y(f(x))$. Поэтому

$$
\# M_{2} \backslash M_{1} \leqslant \sum_{m \geqslant y(f(x))} \frac{f(x)}{m^{2}}=O\left(\frac{f(x)}{y(f(x))}\right) .
$$

Значит, мощность множества $M_{2} \backslash M_{1}$ также не больше остаточного члена в формулировке леммы.

Для оставшихся простых $p$ из множества, определенного в условии леммы 1 , имеem

$$
\frac{p-1}{n}-1=p_{k} m^{(k-1)}
$$

где $p_{k}$ - простое число, $p_{k}>y(f(x))$, а $m^{(k-1)}$ - натуральное такое, что $v\left(m^{(k-1)}\right)=$ $k-1$. Кроме того, по построению $m^{(k-1)}<f(x) / y(f(x))$. Количество указанных в условии простых, за исключением множеств $M_{1}, M_{2}$, не превосходит

$$
\sum_{m \leqslant f(x) / y(f(x)), v(m)=k-1} N\left(p \leqslant x \mid p, \frac{p-1}{n}, \frac{(p-1) / n-1}{m}-\text { простые }\right) .
$$

Каждое слагаемое в этой сумме, соответствующее натуральному $m$, можно представить в виде

$$
N\left(r \leqslant\left[\frac{[(x-1) / n]-1}{m}\right] \mid r, r m+1,(r m+1) n+1-\text { простые }\right) .
$$

При $(m, n+1) \neq 1$ - это 0. Будем считать, что $(m, n+1)=1$.

Для дальнейших оценок используем следующую теорему из метода решета Сельберга.

Tеорема 2 [6; теорема 2.4.6]. Пусть $a_{i}, b_{i} \in \mathbb{Z}, i=1,2, \ldots, s, a_{i} \neq 0,\left(a_{i}, b_{i}\right)=1$. При $i \neq k \quad\left(a_{k}, b_{k}\right) \notin\left\{\left( \pm a_{i}, b_{i}\right),\left( \pm a_{i},-b_{i}\right)\right\}$. Пусть $w(p)$ - число решений сравнения

$$
\left(a_{1} u+b_{1}\right)\left(a_{1} u+b_{1}\right) \cdots\left(a_{s} u+b_{s}\right) \equiv 0(\bmod p),
$$

a

$$
E=\prod_{1 \leqslant i \leqslant s} a_{i} \prod_{1 \leqslant i<k \leqslant s}\left(a_{i} b_{k}-a_{k} b_{i}\right)
$$

Тогда при $N \geqslant 2$ имеет место оценка

$$
N\left(u \leqslant N|| a_{i} u+b_{i} \mid- \text { nростые } \forall i=1, \ldots, s\right)<c(s) \frac{N}{\ln ^{s} N} \prod_{p \mid E}\left(1-\frac{1}{p}\right)^{-(s-w(p))},
$$

где постоянная $c(s)$ зависит только от $s$.

Применим эту теорему при $s=3,\left(a_{i}, b_{i}\right) \in\{(1,0),(m, 1),(m n, n+1)\}$. Тогда $E=m^{2} n(n+1)(m(n+1)-m n)=m^{3} n(n+1)$. Принимая во внимание, что

$$
\left[\frac{[(x-1) / n]-1}{m}\right]<\frac{x}{m n},
$$


имеем следующую оценку выражения (2):

$$
\leqslant C_{1} \frac{x}{m n \ln ^{3}(x /(m n))} \prod_{p \mid E}\left(1-\frac{1}{p}\right)^{-(3-w(p))}
$$

где $w(p)$ - число решений сравнения

$$
x(m x+1)(m n x+n+1) \equiv 0(\bmod p) .
$$

Если $p \mid m$, то $w(p)=1$. Если $p \nmid m, p \mid n$, то $w(p)=2$. Если $p \mid n+1$, а значит, $p \nmid m$, $p \nmid n$, то $w(p)=2$. Поэтому указанную оценку можно продолжить

$$
\begin{aligned}
& \leqslant C_{1} \frac{x}{m n \ln ^{3}(x /(m n))} \prod_{p \mid m}\left(1-\frac{1}{p}\right)^{-2} \prod_{p \mid n(n+1), p \nmid m}\left(1-\frac{1}{p}\right)^{-1} \\
& \leqslant C_{1} \frac{x m^{2} n(n+1)}{m n \ln ^{3}(x /(m n)) \varphi^{2}(m) \varphi(n(n+1))} .
\end{aligned}
$$

Теперь всю сумму (1) можно оценить так:

$$
\leqslant C_{1} \frac{x(n+1)}{\varphi(n(n+1))} \sum_{m \leqslant f(x) / y(f(x)), v(m)=k-1} \frac{m}{\varphi^{2}(m) \ln ^{3}(x /(m n))} .
$$

Поскольку функция $z / y(z)$ возрастает, то эту оценку можно продолжить так:

$$
\begin{aligned}
& <C_{1} \frac{x(n+1)}{\varphi(n(n+1))} \sum_{m \leqslant x /(n y(x / n)), v(m)=k-1} \frac{m}{\varphi^{2}(m) \ln ^{3}(x /(m n))} \\
& <C_{1} \frac{x(n+1)}{\varphi(n(n+1)) \ln ^{3}(y(x / n))} \sum_{m \leqslant x /(n y(x / n)), v(m)=k-1} \frac{m}{\varphi^{2}(m)} \\
& <C_{1} \frac{x(n+1)(\ln \ln (x / n))^{3}}{\varphi(n(n+1)) \ln ^{3}(x / n)} \frac{1}{(k-1) !}\left(\sum_{p<x /(n y(x / n))} \sum_{i=1}^{\infty} \frac{p^{i}}{\varphi^{2}\left(p^{i}\right)}\right)^{k-1} \\
& =C_{1} \frac{x(n+1)(\ln \ln (x / n))^{3}}{\varphi(n(n+1)) \ln ^{3}(x / n)} \\
& \quad \times \frac{1}{(k-1) !}\left(\sum_{p<x /(n y(x / n))}\left(\frac{p}{(p-1)^{2}}+\sum_{i=2}^{\infty} \frac{1}{p^{(i-2)}(p-1)^{2}}\right)\right)^{k-1} .
\end{aligned}
$$

Так как

$$
\sum_{p} \sum_{i=2}^{\infty} \frac{1}{p^{(i-2)}(p-1)^{2}}=\sum_{p} \frac{1}{(p-1)^{2}} \frac{1}{(1-1 / p)}<\infty
$$

a

$$
\sum_{p<A} \frac{p}{(p-1)^{2}}=\sum_{p<A} \frac{1}{p}\left(1+\frac{1}{p-1}\right)^{2}=\sum_{p<A} \frac{1}{p}\left(1+\frac{2}{p-1}+\frac{1}{(p-1)^{2}}\right) \leqslant \ln \ln A+C
$$


для некоторой абсолютной константы $C$, то нашу оценку можно продолжить

$$
\begin{aligned}
& \leqslant C_{1} \frac{x(n+1)(\ln \ln (x / n))^{3}}{\varphi(n(n+1)) \ln ^{3}(x / n)} \frac{1}{(k-1) !}\left(\ln \ln \frac{x}{n y(x / n)}+C_{2}\right)^{k-1} \\
& \leqslant C_{1} \frac{x(n+1)(\ln \ln (x / n))^{3}}{\varphi(n(n+1)) \ln ^{3}(x / n)} \frac{1}{(k-1) !}\left(\ln \ln \frac{x}{n}+C_{2}\right)^{k-1} .
\end{aligned}
$$

Лемма доказана.

ЛЕмма 2 (Эрдёш [6; гл. 5, §7]). Для любой положителъной константы $C$ и любого $\varepsilon>0$ существует $\delta(\varepsilon)>0$ такое, что

$$
\sum_{k \in \mathbb{N}, k \notin[(1-\varepsilon) \ln \ln z,(1+\varepsilon) \ln \ln z]} \frac{(\ln \ln z+C)^{k+2}}{(k-1) !}=O\left(\ln ^{1-\delta(\varepsilon)} z\right)
$$

при $z \rightarrow \infty$.

Применяя эту лемму с заменой $z$ на $x / n$, из леммы 1 суммированием по $k$ получим следующую лемму.

ЛЕмма 3. Для любого $\varepsilon>0$ существует $\delta(\varepsilon)>0$ такое, что для любого $n \in \mathbb{N}$, $n \leqslant x /(y(x))$, выполнено

$$
\begin{aligned}
N(p & \left.\leqslant x \mid \frac{p-1}{n}=q-\text { nростое }, v(q-1) \notin\left[(1-\varepsilon) \ln \ln \frac{x}{n},(1+\varepsilon) \ln \ln \frac{x}{n}\right]\right) \\
& =o\left(\frac{x(n+1)}{\varphi(n(n+1)) \ln ^{2+\delta(\varepsilon)}(x / n)}\right)
\end{aligned}
$$

при $x \rightarrow \infty$, где о $(\cdot)$ не зависит от $n$.

ДокАЗАТЕЛЬСтво теоремы 1. Для доказательства теоремы 1 достаточно установить следующую оценку:

$$
\begin{aligned}
N(p & \left.\leqslant x \mid \begin{array}{c}
\text { существует простое } q>y(x) \text { такое, что } \frac{p-1}{q}=n \in \mathbb{N}, \text { и } \\
v(q-1) \notin\left[(1-\varepsilon) \ln \ln \frac{x}{n},(1+\varepsilon) \ln \ln \frac{x}{n}\right]
\end{array}\right) \\
& =o\left(\frac{x}{\ln ^{1+\delta_{1}} x}\right),
\end{aligned}
$$

где константа $\delta_{1}>0$.

Указанное количество можно оценить по лемме 3 суммированием по $n \leqslant x / y(x)$. Используя оценку Ландау для функции Эйлера, получим для некоторого $\delta>0$ следующую оценку этой величины:

$$
o\left(\sum_{n \leqslant x / y(x)} \frac{x(n+1)}{\varphi(n(n+1)) \ln ^{2+\delta}(x / n)}\right)=o\left(x \ln \ln x \sum_{n \leqslant x / y(x)} \frac{1}{n \ln ^{2+\delta}(x / n)}\right) .
$$

Нетрудно видеть, что при постоянном $s>1$ в силу оценки $y(x)<x$ имеем

$$
\begin{aligned}
\sum_{2 \leqslant n \leqslant x / y(x)} \frac{1}{n \ln ^{s}(x / n)} & \leqslant \int_{1}^{x / y(x)} \frac{d t}{t \ln ^{s}(x / t)} \leqslant \int_{y(x)}^{x} \frac{z x d z}{x z^{2} \ln ^{s} z} \\
& =\int_{y(x)}^{x} \frac{d \ln z}{\ln ^{s} z}=O\left(\frac{1}{\ln ^{s-1} y(x)}\right) .
\end{aligned}
$$


Таким образом, рассматриваемое количество можно оценить так:

$$
o\left(x \ln \ln x \frac{(\ln \ln x)^{1+\delta}}{\ln ^{1+\delta} x}\right)=o\left(\frac{x}{\ln ^{1+\delta_{1}} x}\right) .
$$

Теорема доказана.

Заметим, что теми же средствами могут быть получены аналогичные результаты для всех простых чисел из дерева числа $n$, отстоящих от $n$ на постоянное, т.е. не растущее с ростом $n$, число звеньев. Для получения указанным способом таких оценок для остальных узлов дерева $n$ необходимо в методе Сельберга получить более точную и эффективную зависимость от количества рассматриваемых линейных комбинаций.

\section{СПИСОК ЦИТИРОВАННОЙ ЛИТЕРАТУРЫ}

[1] K. Sakurai, H. Shizuya, "Relationships among the computational powers of breaking discrete log cryptosystems", Advances in cryptology-EUROCRYPT '95 (Saint-Malo, 1995), Lecture Notes in Comput. Sci., 921, Springer, Berlin, 1995, 341-355.

[2] М.А. Черепнёв, "О связи сложностей задач дискретного логарифмирования и Диффи-Хеллмана", Дискр. матем., 8:3 (1996), 22-30.

[3] G. H. Hardy, S. Ramanujan, "The normal number of prime factors of a number $n$ ", $Q . J$. Math., 48 (1917), 76-92.

[4] P. Turán, "On a theorem of Hardy and Ramanujan", J. London Math. Soc. (2), 30 (1929), 93-111.

[5] P. Erdős, "On the normal number of prime factors of $p-1$ and some related problems concerning Euler's $\varphi$-function", Q. J. Math. (Oxford), 6 (1935), 205-213.

[6] К. Прахар, Распределение простых чисел, Мир, М., 1967.

\section{М. А. Черепнёв}

Московский государственный университет им. М. В. Ломоносова

E-mail: ma@atom.ru
Поступило

29.03.2004

Исправленный вариант

04.05.2006 